\title{
Benchmarking of the Dose Planning Method (DPM) Monte Carlo code using electron beams from a racetrack microtron ${ }^{\text {a) }}$
}

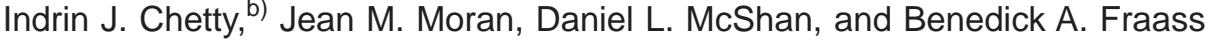 \\ The University of Michigan, Department of Radiation Oncology, Ann Arbor, Michigan 48109-0010 \\ Scott J. Wilderman and Alex F. Bielajew \\ The University of Michigan, Department of Nuclear Engineering, Ann Arbor, Michigan 48109-2104
}

(Received 20 August 2001; accepted for publication 5 April 2002; published 15 May 2002)

\begin{abstract}
A comprehensive set of measurements and calculations has been conducted to investigate the accuracy of the Dose Planning Method (DPM) Monte Carlo code for dose calculations from 10 and $50 \mathrm{MeV}$ scanned electron beams produced from a racetrack microtron. Central axis depth dose measurements and a series of profile scans at various depths were acquired in a water phantom using a Scanditronix type RK ion chamber. Source spatial distributions for the Monte Carlo calculations were reconstructed from in-air ion chamber measurements carried out across the twodimensional beam profile at $100 \mathrm{~cm}$ downstream from the source. The in-air spatial distributions were found to have full width at half maximum of 4.7 and $1.3 \mathrm{~cm}$, at $100 \mathrm{~cm}$ from the source, for the 10 and $50 \mathrm{MeV}$ beams, respectively. Energy spectra for the 10 and $50 \mathrm{MeV}$ beams were determined by simulating the components of the microtron treatment head using the code MCNP4B. DPM calculations are on average within $\pm 2 \%$ agreement with measurement for all depth dose and profile comparisons conducted in this study. The accuracy of the DPM code illustrated in this work suggests that DPM may be used as a valuable tool for electron beam dose calculations. (C) 2002 American Association of Physicists in Medicine. [DOI: 10.1118/1.1481512]
\end{abstract}

Key words: racetrack microtron, Monte Carlo, DPM, ion-chamber measurements

\section{INTRODUCTION}

Ever since the seminal work by Petti et al. ${ }^{1}$ in 1983 , and Mohan et al. ${ }^{2}$ in 1985, who applied the Monte Carlo method to the study of linear accelerators and radiotherapy dose calculations, there has been steady progression in the use of this method in the field of radiation therapy. The literature is currently replete with research from investigators involved in testing and modifying Monte Carlo codes for use in dose calculations. $^{2-11}$ Although the use of the Monte Carlo method has evolved significantly in the field of radiation therapy, the issue of routine treatment planning within a reasonable amount of time (on the order of a few minutes) still remains a concern. Rapid advances in processor technology, however, is bound to soon provide a solution to this limitation; in fact many investigators are currently taking advantage of parallel processing to perform routine Monte Carlo dose calculations. ${ }^{4,5,12}$ The limitation in processing times for Monte Carlo dose calculations has also prompted researchers to improve the efficiency of their Monte Carlo dose engines. Two examples of this are the codes developed by Wang et al. ${ }^{6}$ and Kawrakow et al. ${ }^{7}(\mathrm{VMC}++)$ respectively. Both these codes make use of powerful variance reduction techniques to significantly improve the dose calculation efficiency within the context of a patient-specific CT-based voxelized geometry. ${ }^{6,7}$ A new Monte Carlo code, the Dose Planning Method (DPM) has more recently been developed by Sempau et al. ${ }^{13}$ DPM has also been optimized for radiotherapy dose calculations and employs a robust coupled photon-electron transport scheme that is accurate and efficient. $^{13}$
The focus of the current work was to benchmark the DPM code against a series of measurements conducted using electron beams produced from a racetrack microtron. While the paper by Sempau et al. ${ }^{13}$ established the accuracy of DPM relative to other well-established Monte Carlo codes, such as EGS4/PRESTA and PENELOPE, no comparisons were provided against measurements. Extension of the work by Sempau et al. ${ }^{13}$ for dose calculations in a clinical setting requires testing of the code against standard measurements in a water phantom. The motivation for this work was to benchmark the code under conditions that would provide a test of the transport physics used in the code; the lateral disequilibrium observed with high energy, monoenergetic, pencil-beam electrons in water, for example, poses a challenging test of the physics for any dose computational algorithm. The $50 \mathrm{MeV}$ electron beam used in this study was approximately monoenergetic, with a pencil-beam type spatial distribution (full width at half maximum of $1.3 \mathrm{~cm}$ at $100 \mathrm{~cm}$ from the source). The minimal source modeling requirement and significant lateral electron disequilibrium observed with this type of beam were found to be ideal for evaluating the transport physics used in DPM. In describing the details of this study, this paper will address the following areas: overview of the DPM Monte Carlo code, Monte Carlo simulation of the treatment head for the electron beams from the racetrack microtron, the measurement setup, the Monte Carlo source description and scoring parameters, and finally, water phantom dose comparisons between measurements and calculations. 
TABLE I. Description of the treatment head of the Scanditronix Racetrack Microtron (Scanditronix, Uppsala, Sweden) for the 10 and $50 \mathrm{MeV}$ uncollimated electron beams. Shown are the component composite materials, and the corresponding density-weighted thicknesses in $\mathrm{g} / \mathrm{cm}^{2}$. The data presented here are based on information provided by Scanditronix engineers. Simulation of the various components was conducted using the Monte Carlo method to determine the electron energy spectra.

\begin{tabular}{lcc}
\hline \hline \multicolumn{1}{c}{ Component } & Material & $\begin{array}{c}\text { Density-weighted } \\
\text { thickness }\left(\mathrm{g} / \mathrm{cm}^{2}\right.\end{array}$ \\
\hline $\begin{array}{l}\text { Entrance window } \\
\text { Ion chamber }\end{array}$ & $\begin{array}{c}\text { Beryllium } \\
\text { Layers of gold and } \\
\text { polyamide }\left(\mathrm{CH}_{2}\right)\end{array}$ & $\begin{array}{c}4.63 \times 10^{-2} \\
0.103\end{array}$ \\
Mirror & Kapton & $1.70 \times 10^{-3}$ \\
Exit window & Mylar & $2.40 \times 10^{-3}$ \\
Medium within & Helium & $7.70 \times 10^{-3}$ \\
treatment head & & \\
& & Total $=0.161$ \\
\hline \hline
\end{tabular}

\section{MATERIALS AND METHODS}

\section{A. Overview of the DPM Monte Carlo Code}

The Dose Planning Method (DPMv1.0) Monte Carlo code has been developed by Sempau et al. ${ }^{13}$ for radiotherapy treatment planning dose calculations. DPM is capable of calculating the dose in a CT-based, patient-specific, voxel-based geometry and uses an accurate and efficient coupled electron-photon transport model. Electron transport within DPM uses a condensed history model that is based on a "mixed" transport scheme for energy losses, with analog transport for large energy transfers, and the continuous slowing down approximation (CSDA) used for small energy losses. ${ }^{13}$ Electron multiple scattering is based upon the Kawrakow-Bielajew formalism, which is a robust implementation of the Goudsmit-Saunderson theory for angular sampling of charged particles. ${ }^{13}$ The point-to-point transport of charged particles in a medium uses a "random-hinge" scheme originally developed in the PENELOPE code but adapted to better handle energy losses over large electron steps. ${ }^{13}$ Photons are transported using the Woodcock tracking method which eliminates the inefficient boundary tracking process. ${ }^{13}$ For a detailed discussion of the photon and electron transport physics and other features contained in the DPM Monte Carlo code, the interested reader is referred to the paper by Sempau et al. ${ }^{13}$

\section{B. Treatment head description for the racetrack microtron electron beams and Monte Carlo simulation}

The Scanditronix racetrack microtron (Scanditronix, Uppsala, Sweden) was chosen for this study due to its simple treatment head design, the ability to deliver electron beams without scattering foils or beam collimators, and the range of energies up to $50 \mathrm{MeV} .{ }^{14}$ The treatment head components along with their composite material types and densityweighted thicknesses are presented in Table I. Information in Table I is based upon information provided by Scanditronix engineers. The entire component thickness is approximately $0.2 \mathrm{~g} / \mathrm{cm}^{2}$, which minimizes energy losses in the treatment head. In addition, the entire treatment head, from the vacuum window to the exit window, is filled with helium. Karlsson et al.,${ }^{14}$ in their paper on electron beam characteristics of the $50 \mathrm{MeV}$ racetrack microtron, point out that the $80 \%-20 \%$ penumbra is reduced by a factor of 2 when the air-filled treatment head is replaced with helium; this is due to the much lower linear scattering power in helium. The modified treatment head design results in a $50 \mathrm{MeV}$ beam that is nearly monoenergetic, and has a significantly smaller angular electron spread relative to other accelerators. ${ }^{14}$

The energy spectra of the 10 and $50 \mathrm{MeV}$ electron beams were calculated by Monte Carlo simulation of the treatment head components using the code MCNP4B. ${ }^{15}$ The scoring plane consisted of concentric cylinders, each with radius 2 $\mathrm{mm}$ greater than the previous and extending from the beam central axis to $8 \mathrm{~cm}$ radially outward from the central axis. The scoring plane was situated $100 \mathrm{~cm}$ downstream from the source, in air, with each scoring cylinder spanning a volume with depth $2 \mathrm{~mm}$. The MCNP F4 tally was used to score the energy fluence. The F4 tally uses a track length estimate of the particle fluence based on the track length of each particle through the cell volume. ${ }^{15}$ The F4 tally was chosen since it has been found to be a reliable estimate of fluence, given that there are many tracks in a cell and hence many contributions to this tally. ${ }^{15}$ This tally has also been used by other investigators for scoring fluence. ${ }^{16}$ To obtain the incident electron energies, a trial and error method was used whereby each spectral distribution was calibrated against the corresponding central axis depth dose curve. This is a standard method of determining the incident electron energies and has been used by many other investigators. ${ }^{10,17,18}$ For this work, the starting, monoenergetic electron energies were determined to be 10.65 and $50.0 \mathrm{MeV}$ for the 10 and $50 \mathrm{MeV}$ electron beams, respectively.

\section{Measurement setup}

The experimental setup consisted of a series of ionchamber measurements acquired in air as well as within a water phantom of dimensions $40 \times 40 \times 40 \mathrm{~cm}^{3}$. All measurements were conducted using a Scanditronix Type RK 83-05 ion chamber with an air-cavity volume of $0.12 \mathrm{~cm}^{3}$ and a 2 $\mathrm{mm}$ inner radius. Central axis and off-axes "in-air" transverse profile ( $x$ axis) scans were taken for the $10 \mathrm{MeV}$ beam, extending from -6.4 to $6.4 \mathrm{~cm}$ in the $x$ axis, and spanning a region from -6.4 to $6.4 \mathrm{~cm}$ in the $y$ axis in $2 \mathrm{~mm}$ increments. Transverse "in-air" scans were acquired for the 50 $\mathrm{MeV}$ beam extending from -2.4 to $2.4 \mathrm{~cm}$ in the $x$ axis, and spanning a region from -2.4 to $2.4 \mathrm{~cm}$ in the $y$ axis in $2 \mathrm{~mm}$ increments. The total number of transverse "in-air" scans acquired at $100 \mathrm{~cm}$ downstream from the source was therefore 65 for the $10 \mathrm{MeV}$ beam and 25 for the $50 \mathrm{MeV}$ beam. Central axis depth dose and profiles were measured at 100 $\mathrm{cm}$ SSD within the water phantom. Profile scans were acquired along the central axis (along the transverse, $x$ axis) at the depths of $0.5 \mathrm{~cm}$ and $d_{\max }$, as well as the $90 \%, 50 \%$, and $20 \%$ isodose regions. 


\section{Monte Carlo source description and scoring parameters}

The Monte Carlo source description typically requires specification of the position, energy, and angle for each starting particle. A two-dimensional source spatial distribution was reconstructed from the "in-air" profile measurements for sampling the source particle's position. The radial fluence distribution was extracted from the transverse profilesacquiring these scans in $2 \mathrm{~mm}$ increments allowed reconstruction of a finely sampled fluence matrix. For the $50 \mathrm{MeV}$ beam, a monoenergetic $50.0 \mathrm{MeV}$ electron source was used, while for the $10 \mathrm{MeV}$ beam the electron's energy was calculated as a function of position within the sampling plane. The source particle's angle was calculated using a point source $\left(1 / R^{2}\right)$ divergence. Based on the agreement with measurements (see Sec. III), the point-source approximation provides an adequate description of the electron beam angular spread.

Calculations using the DPM Monte Carlo code were performed using a simulated cubic water phantom with side 40 $\mathrm{cm}$. A scoring voxel with dimensions $2 \mathrm{~mm} \times 2 \mathrm{~mm} \times 2 \mathrm{~mm}$ was used for all calculations. The low energy electron and photon cutoffs were 200 and $50 \mathrm{keV}$, respectively, and the DPM step size was set at $2 \mathrm{~mm}$ for both 10 and $50 \mathrm{MeV}$ comparisons. The influence of step size on electron beam dose distributions and the electron physics modifications to handle transport at larger step sizes is discussed at length in the paper by Sempau et al. ${ }^{13}$

\section{RESULTS AND DISCUSSION}

Figures 1(a) and 1(b) show the percentage electron fluence averaged over the scoring plane as a function of energy for the 10 and $50 \mathrm{MeV}$ electron beams, respectively. Each energy bin in Fig. 1(a) has a width of $0.15 \mathrm{MeV}$. It is seen that $96 \%$ of the electron fluence is accounted for in the energy region from 9.9 to $10.65 \mathrm{MeV}$. Although not explicitly illustrated on the plot, simulation results indicate that approximately $3 \%$ of electrons have energies in the range from 0 to $9 \mathrm{MeV}$. Figure 1(b) shows that $98 \%$ of electrons have energies in the range from 49.8 to $50.0 \mathrm{MeV}$; each bin here has a width of $0.2 \mathrm{MeV}$. The remaining $2 \%$ of electrons occupy energies in the region from 0 to $49.8 \mathrm{MeV}$. These results suggest that the energy losses due to scattering in the microtron treatment head are minimal for the $50 \mathrm{MeV}$ beam.

Figures 2(a) and 2(b) illustrate the central axis depth dose curves for the 10 and $50 \mathrm{MeV}$ uncollimated electron beams respectively, normalized to the maximum dose. Plots of the percent differences between DPM and measurements as well as the $1 \sigma$ Monte Carlo statistical uncertainty are shown in Fig. 2(c). The RMS average differences between measurements and DPM are $0.4 \%$ and $0.6 \%$ for the 10 and $50 \mathrm{MeV}$ electron beams, respectively, which are well within the $2 \%$ acceptability criteria suggested by Van Dyk et al. ${ }^{19}$ for electron beams along the central ray in homogeneous media. Figures 3(a)-7(a) represent relative profile dose comparisons for the 10 and $50 \mathrm{MeV}$ beams at depths of $0.5 \mathrm{~cm}$ and $d_{\max }$, and the $90 \%, 50 \%$, and $20 \%$ isodose regions, respectively. The $d_{\max }, 90 \%, 50 \%$, and $20 \%$ isodose regions correspond to
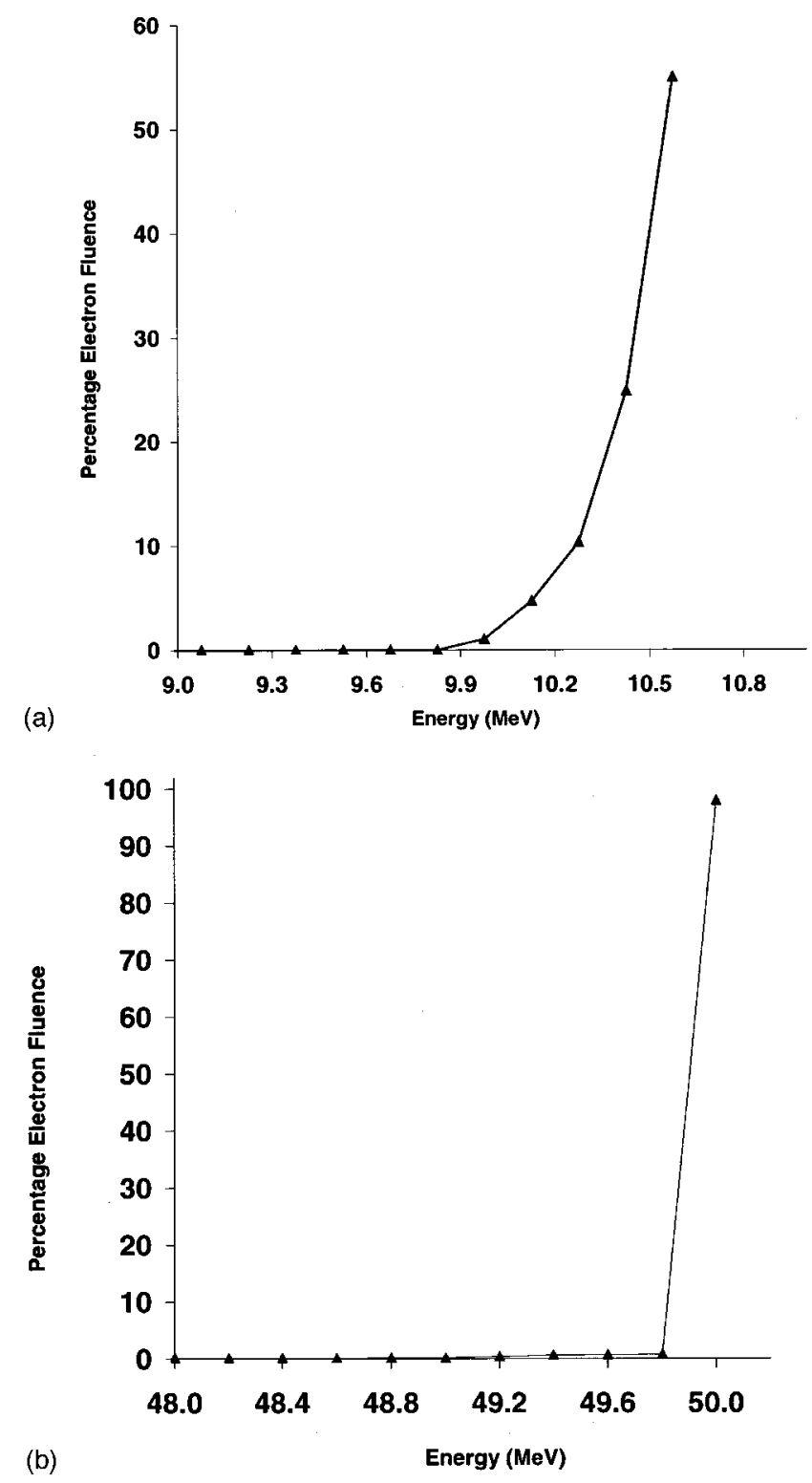

FIG. 1. (a) Percentage electron fluence as a function of energy for the 10 $\mathrm{MeV}$ electron beam, from the MCNP Monte Carlo treatment head simulation of the racetrack microtron. The simulation was conducted starting with 10.65 MeV monoenergetic electrons. The electron fluence is averaged over the concentric cylinders extending from the central axis to a distance of 6 $\mathrm{cm}$ radially outward. $97 \%$ of the electrons occupy energies in the range from 9.9 to $10.65 \mathrm{MeV}$. $3 \%$ of the electrons reside in energy bins from 0 to $9 \mathrm{MeV}$ (not shown on plot). (b) Percentage electron fluence as a function of energy for the $50 \mathrm{MeV}$ electron beam, from the Monte Carlo treatment head simulation of the racetrack microtron. The simulation was conducted starting with $50.0 \mathrm{MeV}$ monoenergetic electrons. The electron fluence is averaged over the concentric cylinders extending from the central axis to a distance of $4 \mathrm{~cm}$ radially outward. The majority of the electrons (98\%) occupy energies in the range from 49.8 to $50.0 \mathrm{MeV}$. A small percentage, $2 \%$, of electrons reside in energy bins from 0 to $49.8 \mathrm{MeV}$.

respective depths of $2.2,3.1,4.3$, and $4.9 \mathrm{~cm}$ for the $10 \mathrm{MeV}$ beam and 1.0, 3.6, 7.6, and $11.8 \mathrm{~cm}$ for the $50 \mathrm{MeV}$ beam. All profiles in this study are normalized to the maximum central ray point dose. The corresponding percentage differences between DPM and measurements are illustrated in Figs. 3(b)-7(b). The difference plots were calculated as fol- 

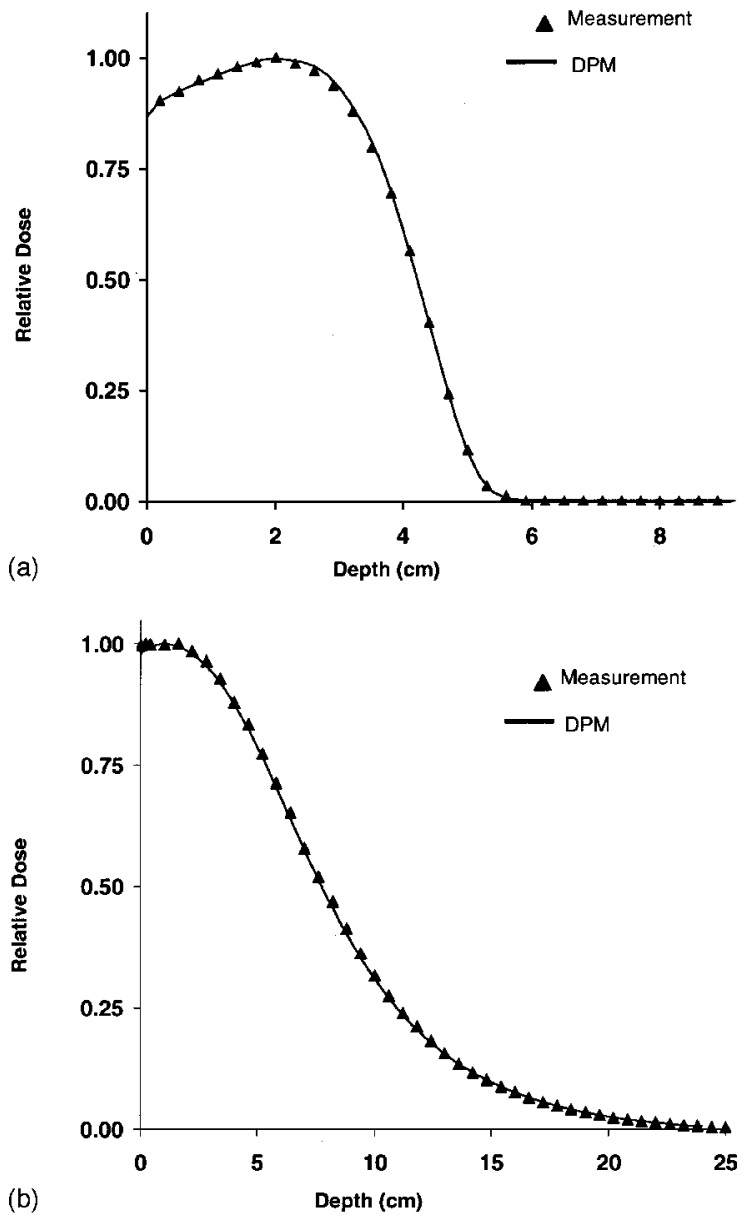

(b)

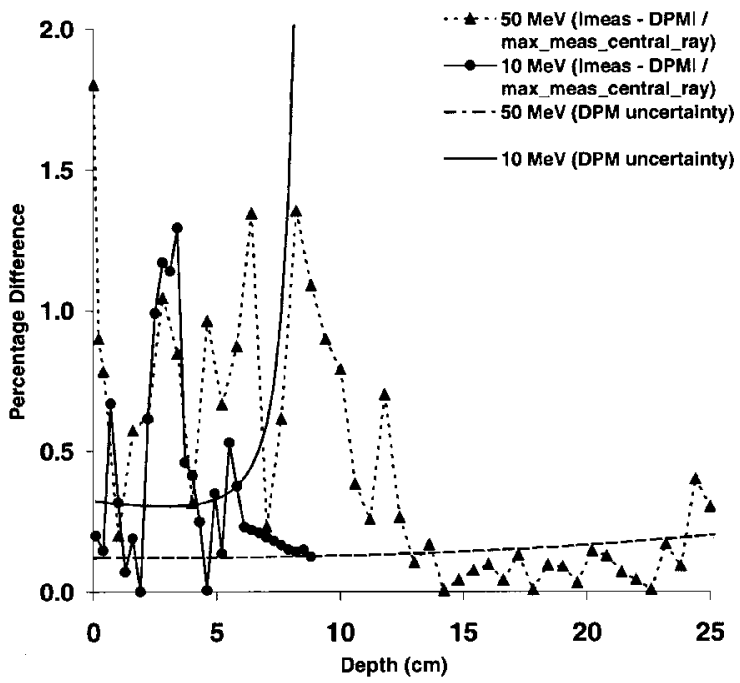

Fig. 2. (a) The $10 \mathrm{MeV}$ central axis depth dose comparison. Measurements are shown with markers, and DPM in the solid line. Both curves are normalized to $d_{\max }$. DPM calculated points represent the center of voxel values. The $1 \sigma$ Monte Carlo uncertainty averaged over all calculation points is $0.5 \%$. (b) The $50 \mathrm{MeV}$ central axis depth dose comparison. Measurements are shown with markers, and DPM in the solid line. Both curves are normalized to $d_{\max }$. DPM calculated points represent the center of voxel values. The $1 \sigma$ Monte Carlo uncertainty averaged over all calculation points is $0.5 \%$. (c) Percent differences between calculations and measurements and the $1 \sigma$ Monte Carlo statistical uncertainty for the depth dose comparisons presented in Figs. 2(a) and 2(b). Percent differences were calculated by taking the absolute value of the difference between measured and calculated values. RMS average differences between measurements and calculations are $0.4 \%(10 \mathrm{MeV})$ and $0.6 \%(50 \mathrm{MeV})$.
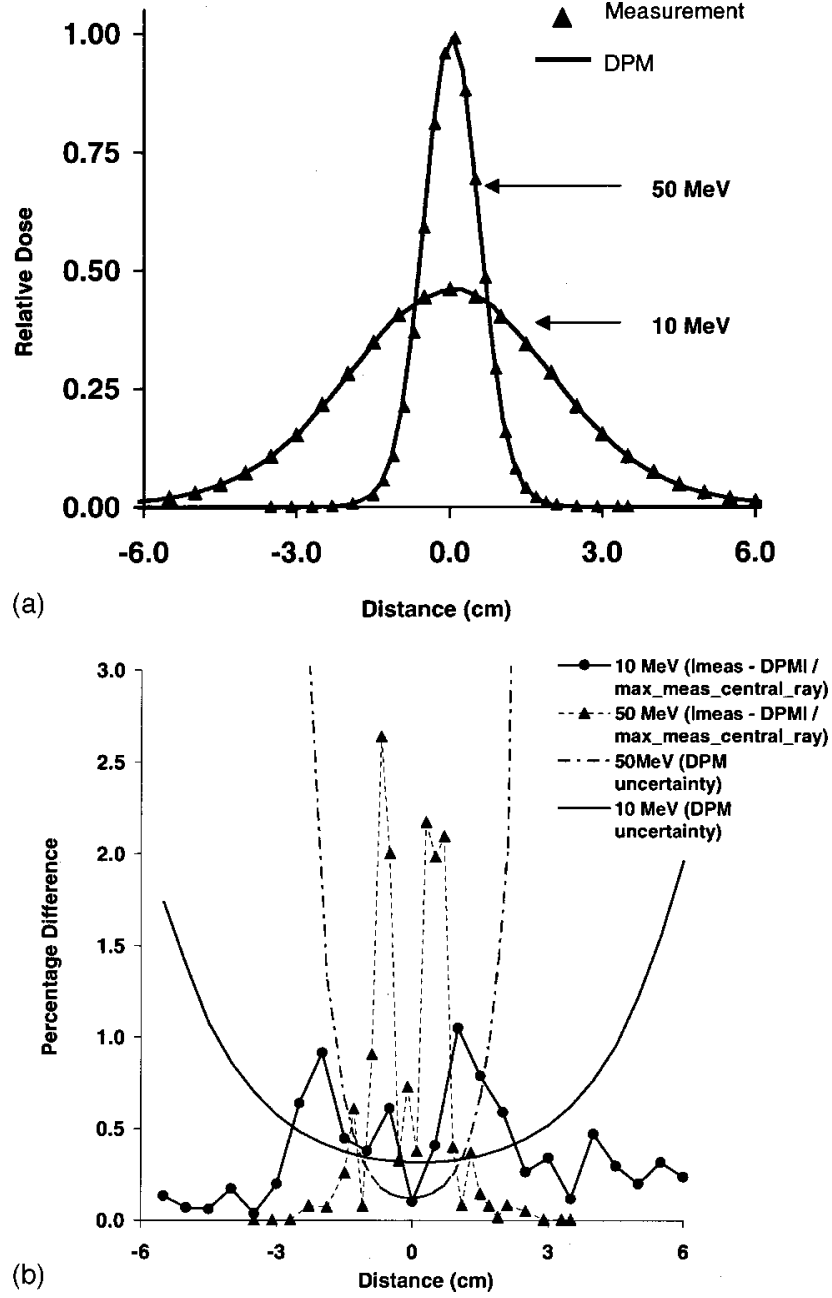

FIG. 3. (a) Central axis profile comparisons at a depth of $0.5 \mathrm{~cm}$ for the 10 and $50 \mathrm{MeV}$ electron beams. Measurements are shown in the triangular marker and DPM in the solid line. DPM calculated points represent the center of voxel values. Curves for each energy are normalized to the respective maximum central ray point doses. The $10 \mathrm{MeV}$ comparison also includes a 0.5 scaling factor for ease of illustration. The $1 \sigma$ Monte Carlo uncertainty averaged over all calculation points is $0.8 \%$ and $1.0 \%$ for the 10 and $50 \mathrm{MeV}$ electron beams, respectively. (b) Percent differences between calculations and measurements and the $1 \sigma$ Monte Carlo statistical uncertainty for the $0.5 \mathrm{~cm}$ depth profile comparison presented in (a). Percent differences were calculated by taking the absolute value of the difference between measured and calculated values. RMS average differences between measurements and calculations are $0.5 \%(10 \mathrm{MeV})$ and $1.0 \%(50 \mathrm{MeV})$.

lows: (|measured value-calculated value $\mid \times 100)$ /maximum central ray point value. Although the average agreement is within $\pm 1.5 \%$ for each profile comparison, maximum differences on the order of $2 \%-3 \%$ are noted in each of the 50 $\mathrm{MeV}$ electron beam profiles. From the Monte Carlo uncertainty graphs for each profile, it is clear that these differences are not due to statistical uncertainty in the calculated points. The cause of these differences is not clear, however, we suspect that the differences are due to a combination of measurement error as well as errors caused by interpolation and smoothing during reconstruction of the fluence map from in-air fluence measurements.

While the calculated $10 \mathrm{MeV}$ profile doses are in better overall agreement with measurements compared with those 

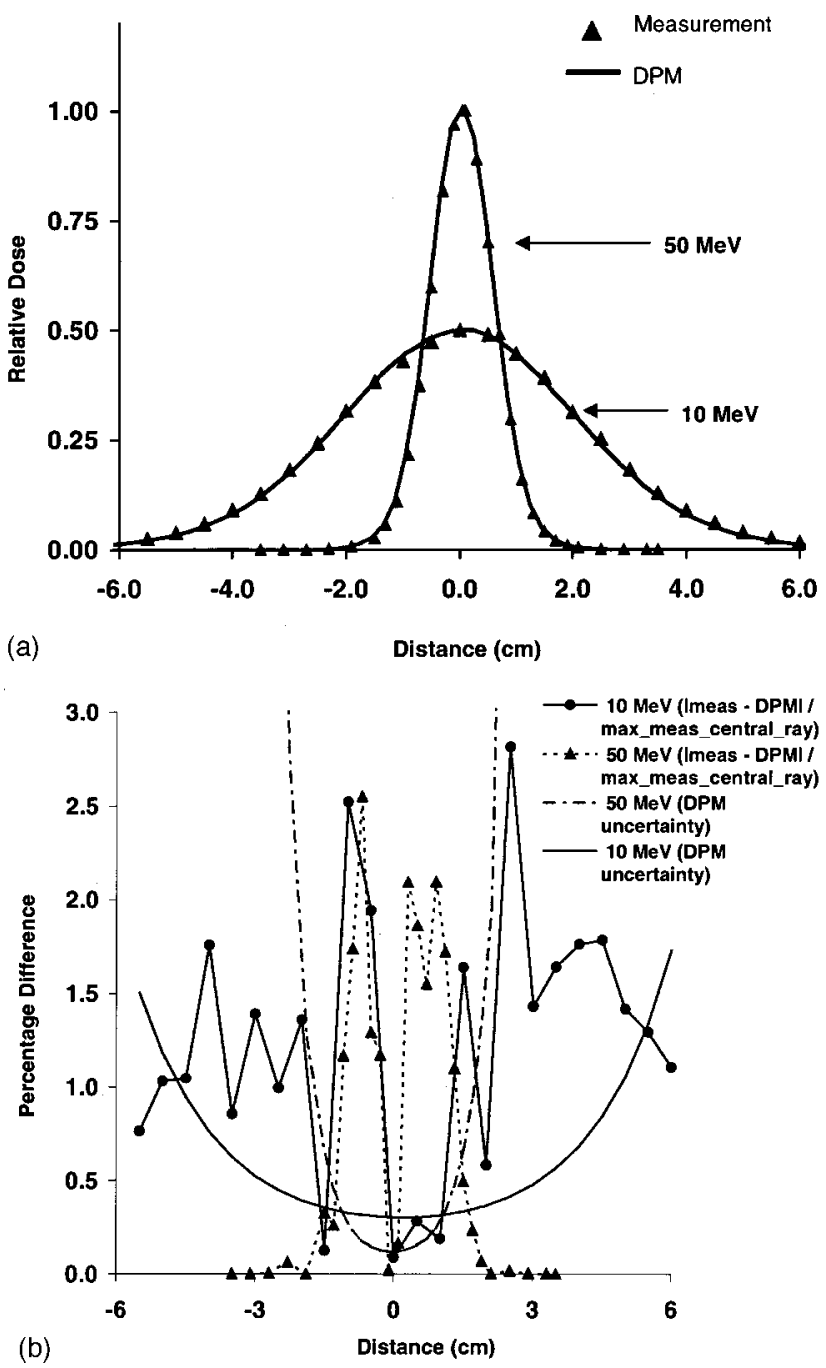

FIG. 4. (a) Central axis profile comparisons at depths of maximum dose for the 10 and $50 \mathrm{MeV}$ electron beams. The $d_{\max }$ depths are 2.2 and $1.0 \mathrm{~cm}$ for the 10 and $50 \mathrm{MeV}$ beams, respectively. Measurements are shown in the triangular marker and DPM in the solid line. DPM calculated points represent the center of voxel values. Curves for each energy are normalized to the respective maximum central ray point doses; the normalization factor is 1.0 at these depths. The $10 \mathrm{MeV}$ comparison also includes a 0.5 scaling factor for ease of illustration. The $1 \sigma$ Monte Carlo uncertainty averaged over all calculation points is $0.7 \%$ and $0.9 \%$ for the 10 and $50 \mathrm{MeV}$ electron beams, respectively. (b). Percent differences between calculations and measurements and the $1 \sigma$ Monte Carlo statistical uncertainty for the profile comparison at $d_{\max }$, illustrated in (a). Percent differences were calculated by taking the absolute value of the difference between measured and calculated values. RMS average differences between measurements and calculations are $1.4 \%(10 \mathrm{MeV})$ and $1.1 \%(50 \mathrm{MeV})$.

at $50 \mathrm{MeV}$, maximum differences in the range of $2 \%-3 \%$ are also seen for the $10 \mathrm{MeV}$ profiles. In particular, maximum differences of $2.8 \%, 2.2 \%$, and $2.0 \%$ are present for the profiles acquired at the $d_{\max }$ [Fig. 4(b)], 90\% [Fig. 5(b)], and $50 \%$ [Fig. 6(c)] isodose regions, respectively. As the statistical uncertainty in these points is much less than the differences versus measurement, the differences noted here are not due to statistical issues. The disagreement may be attributed to uncertainties in the measurements as well systematic errors introduced during the reconstruction of the fluence map.

In their paper on commissioning and quality assurance of
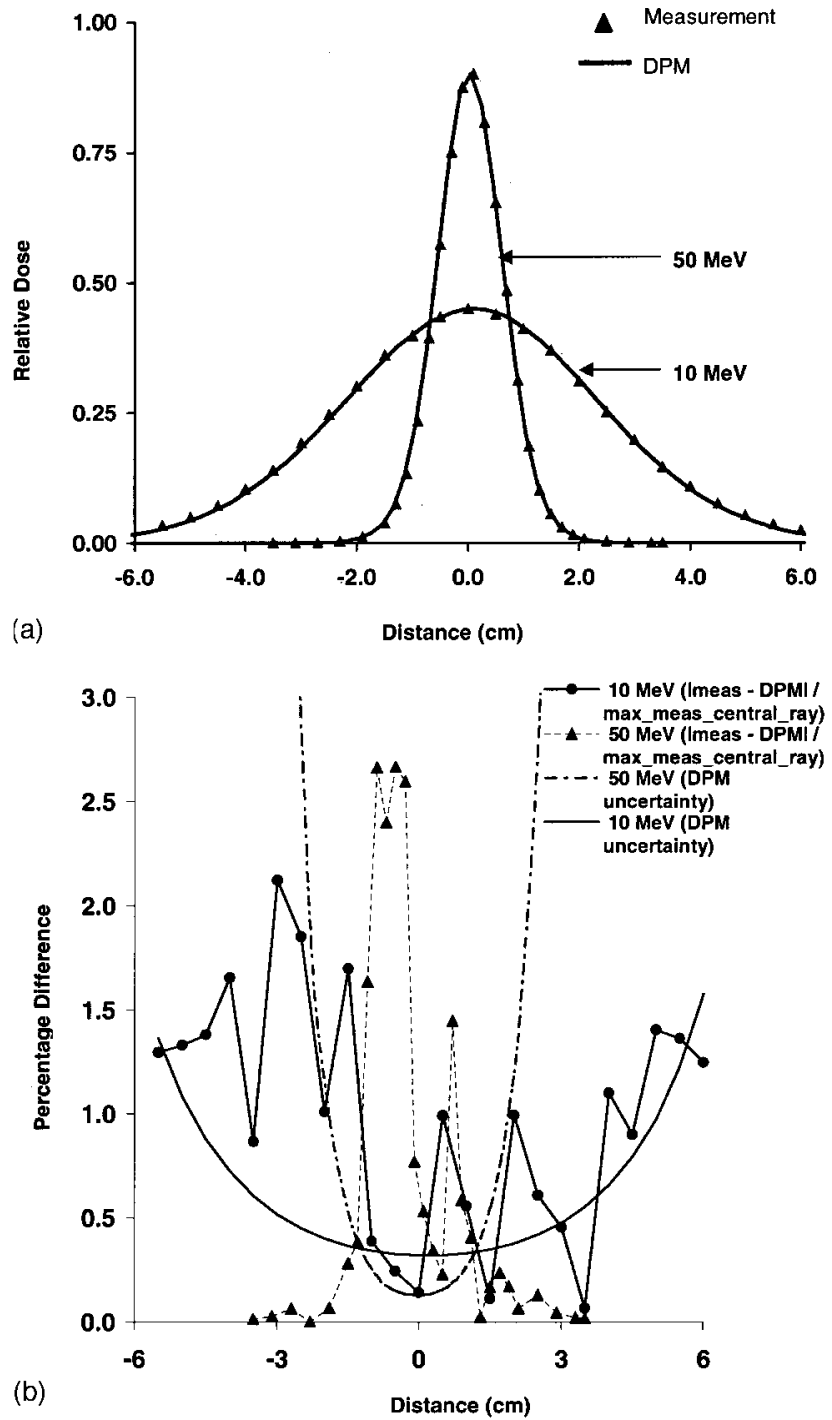

FIG. 5. (a) Central axis profile comparisons at the $90 \%$ isodose region for the 10 and $50 \mathrm{MeV}$ electron beams. The $90 \%$ isodose region corresponds to depths of 3.1 and $3.6 \mathrm{~cm}$ for the 10 and $50 \mathrm{MeV}$ beams, respectively. Measurements are shown in the triangular marker and DPM in the solid line. DPM calculated points represent the center of voxel values. Curves for each energy are normalized to the respective maximum central ray point doses. The $10 \mathrm{MeV}$ comparison also includes a 0.5 scaling factor for ease of illustration. The $1 \sigma$ Monte Carlo uncertainty averaged over all calculation points is $0.6 \%$ and $0.8 \%$ for the 10 and $50 \mathrm{MeV}$ electron beams, respectively. (b) Percent differences between calculations and measurements and the $1 \sigma$ Monte Carlo statistical uncertainty for the profile comparison at the $90 \%$ isodose region, illustrated in (a). Percent differences were calculated by taking the absolute value of the difference between measured and calculated values. RMS average differences between measurements and calculations are $1.1 \%(10 \mathrm{MeV})$ and $1.1 \%(50 \mathrm{MeV})$.

treatment planning computers, Van Dyk et al. ${ }^{19}$ provide criteria of acceptability for electron beam dose calculations versus measurements in homogeneous media. Specifically, the criterion in the high dose region-low dose gradient is $4 \%$, while that in the large dose gradients $(>30 \% / \mathrm{cm})$ is $4 \mathrm{~mm}$. Although DPM calculations in this work are found to be up to $3 \%$ different from measurement at given points, we find that the agreement is nonetheless well within the $4 \mathrm{~mm}$ acceptability criterion recommended by Van Dyk et al. ${ }^{19}$ The 4 

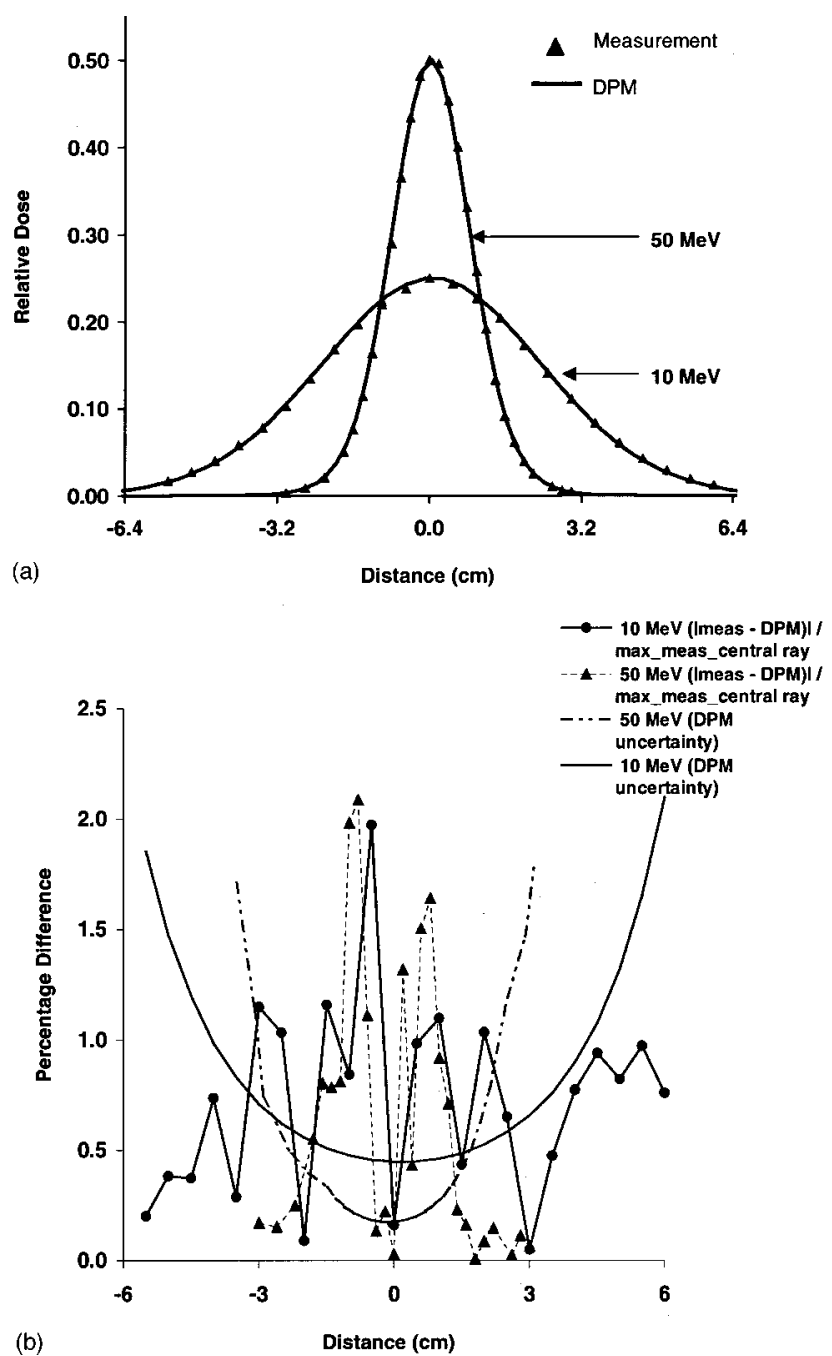

Fig. 6. (a) Central axis profile comparisons at the $50 \%$ isodose region for the 10 and $50 \mathrm{MeV}$ electron beams. The $50 \%$ isodose region corresponds to depths of 4.3 and $7.6 \mathrm{~cm}$ for the 10 and $50 \mathrm{MeV}$ beams, respectively. Measurements are shown in the triangular marker and DPM in the solid line. DPM calculated points represent the center of voxel values. Curves for each energy are normalized to the respective maximum central ray point doses. The $10 \mathrm{MeV}$ comparison also includes a 0.5 scaling factor for ease of illustration. The $1 \sigma$ Monte Carlo uncertainty averaged over all calculation points is $0.9 \%$ and $0.5 \%$ for the 10 and $50 \mathrm{MeV}$ electron beams, respectively. (b) Percent differences between calculations and measurements and the $1 \sigma$ Monte Carlo statistical uncertainty for the profile comparison at the $50 \%$ isodose region, illustrated in (a). Percent differences were calculated by taking the absolute value of the difference between measured and calculated values. RMS average differences between measurements and calculations are $0.8 \%(10 \mathrm{MeV})$ and $0.9 \%(50 \mathrm{MeV})$.

$\mathrm{mm}$ profile criterion is appropriate for comparison purposes in this study given that all points within the $10 / 50 \mathrm{MeV}$ profiles fall within the high-dose gradient region.

\section{CONCLUSION}

This investigation has shown that the DPM Monte Carlo code is capable of calculating accurately the dose to a water phantom from 10 and $50 \mathrm{MeV}$ electron beams. A series of depth dose and profile ion chamber measurements within a water tank has been acquired using minimally scattered electron beams produced from a racetrack microtron, which has a
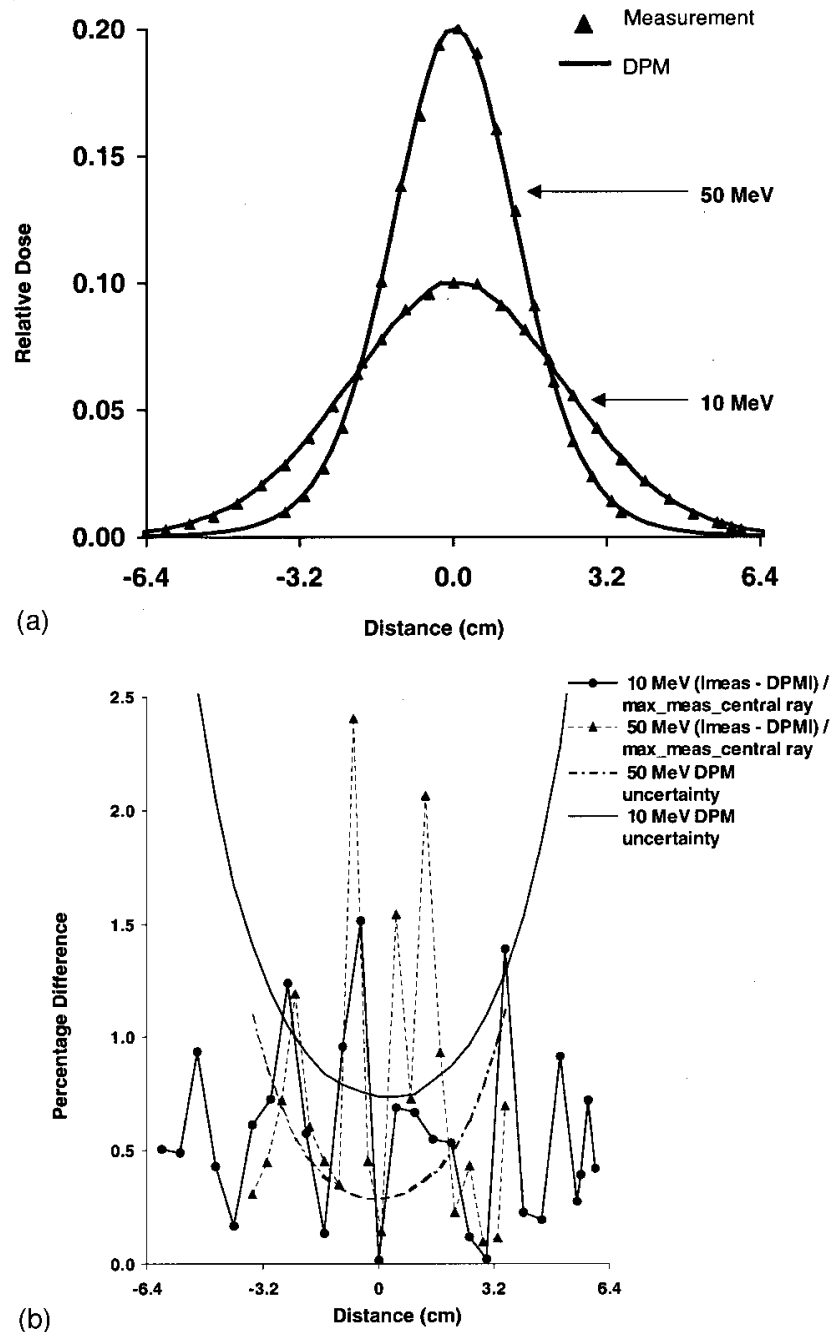

FIG. 7. (a) Central axis profile comparisons at the $20 \%$ depth-isodose region for the 10 and $50 \mathrm{MeV}$ electron beams. The $20 \%$ isodose region corresponds to depths of 4.9 and $11.8 \mathrm{~cm}$ for the 10 and $50 \mathrm{MeV}$ beams, respectively. Measurements are shown in the triangular marker and DPM in the solid line. DPM calculated points represent the center of voxel values. Curves for each energy are normalized to the respective maximum central ray point doses. The $10 \mathrm{MeV}$ comparison also includes a 0.5 scaling factor for ease of illustration. The $1 \sigma$ Monte Carlo uncertainty averaged over all calculation points is $1.5 \%$ and $0.4 \%$ for the 10 and $50 \mathrm{MeV}$ electron beams, respectively. (b) Percent differences between calculations and measurements and the $1 \sigma$ Monte Carlo statistical uncertainty for the profile comparison at the $20 \%$ isodose region, illustrated in (a). Percent differences were calculated by taking the absolute value of the difference between measured and calculated values. RMS average differences between measurements and calculations are $0.7 \%(10 \mathrm{MeV})$ and $0.9 \%(50 \mathrm{MeV})$.

relatively uncomplicated treatment head design (i.e., without scattering foils and collimators). The agreement between DPM measurements is, on average, well within $\pm 2 \%$ for 10 and $50 \mathrm{MeV}$ central axis depth dose and profile comparisons, suggesting that the DPM-electron transport model is accurate in homogeneous situations. A future paper will focus on benchmarking the electron transport model in heterogeneous geometries, where lateral disequilibrium effects are emphasized. 


\section{ACKNOWLEDGMENTS}

This work has been supported in part by NIH Grant No. P01-CA59827. We would like to thank Lianyan Liu, Tim Nurushev, and Shigeru Yokoyama for their valuable help with this project. We would also like to thank Anders Larsson of Scanditronix, Uppsala, Sweden, for providing details on the treatment head design of the racetrack microtron. One of the authors (A.F.B.) gratefully acknowledges the support of ADAC laboratories, Milpitas, CA, for DPM development.

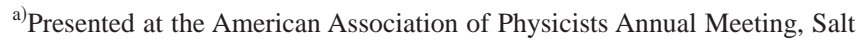
Lake City, UT 2001.

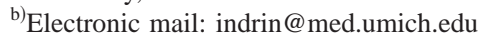

${ }^{1}$ P. L. Petti, M. S. Goodman, T. A. Gabriel, and R. Mohan, "Investigation of buildup dose from electron contamination of clinical photon beams," Med. Phys. 10, 18-25 (1983).

${ }^{2}$ R. Mohan, C. Chui, and L. Lidofsky, "Energy and angular distributions of photons from medical linear accelerators," Med. Phys. 12, 592-597 (1985).

${ }^{3}$ A. E. Schach von Wittenau, L. J. Cox, P. M. Bergstrom, W. P. Chandler, and C. L. Hartmann-Siantar, "Correlated histogram representation of Monte Carlo derived medical accelerator photon output phase space," Med. Phys. 26, 1196-1212 (2000).

${ }^{4}$ C. M. Ma, A. Kapur, T. Pawlicki, D. Findley, S. Brain, and K. Forster, "Clinical implementation of a Monte Carlo treatment planning system," Med. Phys. 26, 2133-2143 (1999).

${ }^{5}$ J. V. Siebers, P. J. Keall, J. O. Kim, and R. Mohan, "Performance benchmarks of the MCV Monte Carlo system," in Proceedings of the XIIIth International Conference on the Use of Computers in Radiation Therapy, edited by W. Schlegel and T. Bortfeld (Springer, Berlin, 2000), pp. 129131.

${ }^{6}$ L. Wang, C. S. Chui, and M. Lovelock, “A patient-specific Monte Carlo dose-calculation method for photon beams," Med. Phys. 25, 867-878 (1998).

${ }^{7}$ I. Kawrakow, M. Fippel, and K. Friedrich, “3D electron dose calculation using a Voxel based Monte Carlo algorithm (VMC)," Med. Phys. 23, 445-457 (1996).

${ }^{8}$ J. Deng, S. B. Jiang, J. Li, T. Pawlicki, and C. M. Ma, "Photon beam characterization and modeling for Monte Carlo treatment planning," Phys. Med. Biol. 45, 411-427 (2000).

${ }^{9}$ C. M. Ma, P. Reckwerdt, M. Holmes, D. W. O. Rogers, and B. Geiser, “DOSXYZ Users Manual,” NRCC Report No. PIRS 509B(rev C), 1998.

${ }^{10}$ J. J. DeMarco, T. D. Solberg, and J. B. Smathers, "A CT-based Monte Carlo dosimetry tool for radiotherapy treatment planning and analysis," Med. Phys. 25, 1-11 (1998).

${ }^{11}$ M. K. Fix, M. Stampanoni, P. Manser, E. J. Born, R. Mini, and P. Ruegsegger, "A multiple source model for $6 \mathrm{MV}$ photon beam dose calculations," Phys. Med. Biol. 46, 1407-1428 (2001).

${ }^{12}$ I. Chetty, J. J. DeMarco, and T. D. Solberg, "A virtual source model for Monte Carlo modeling of arbitrary intensity distributions," Med. Phys. 27, 166-172 (2000).

${ }^{13}$ J. Sempau, S. J. Wilderman, and A. F. Bielajew, "DPM, a fast, accurate Monte Carlo code optimized for photon and electron radiotherapy treatment planning dose calculations," Phys. Med. Biol. 45, 2263-2291 (2000).

${ }^{14}$ M. Karlsson, H. Nystrom, and H. Svensson, "Electron beam characteristics of the 50-MeV racetrack microtron," Med. Phys. 19, 307-315 (1992).

${ }^{15}$ J. F. Briesmeister, "MCNPTM-A general Monte Carlo N-Particle transport code," Los Alamos National Laboratory Report No. LA-12625-M, 1997.

${ }^{16}$ J. J. DeMarco, T. D. Solberg, R. E. Wallace, and J. B. Smathers, "A verification of the Monte Carlo code MCNP for thick target bremsstrahlung calculations," Med. Phys. 22, 11-16 (1995).

${ }^{17}$ D. M. J. Lovelock, C. S. Chui, and R. Mohan, "A Monte Carlo model of photon beams used in radiation therapy," Med. Phys. 22, 1387-1394 (1995).

${ }^{18}$ D. W. O. Rogers, D. A. Faddegon, G. X. Ding, C. M. Ma, J. We, and T. R. Mackie, "BEAM: A Monte Carlo code to simulate radiotherapy treatment units," Med. Phys. 22, 503-524 (1995).

${ }^{19}$ J. Van Dyke, R. B. Barnett, J. E. Cygler, and P. C. Shragge, "Commissioning and quality assurance of treatment planning computers," Int. J. Radiat. Oncol., Biol., Phys. 26, 261-273 (1992). 